\title{
Generalized van der Waals theory for phase behavior of two-dimensional nematic liquid crystals. II. Phase coexistence and adsorption
}

\author{
Ezequiel Rodolfo Soulé \\ Institute of Materials Science and Technology (INTEMA), University of Mar del Plata and National Research Council (CONICET), \\ J. B. Justo 4302, 7600 Mar del Plata, Argentina
}

(Received 16 September 2020; accepted 30 November 2020; published 21 December 2020)

\begin{abstract}
Adsorption of asymmetric particles or molecules into monolayers is important for many biological and technologically relevant physical systems. In-plane ordering can drastically affect adsorption and phase behavior. In this work, a generalized van der Waals theory previously developed [M. V. Zonta and E. R. Soulé, Phys. Rev. E 100, 062703 (2019)] is used to calculated phase behavior and adsorption isotherms in a system of hard-core rodlike particles with in-plane nematic order, as a function of the model parameters (aspect ratio $L / B$, isotropic and anisotropic interaction parameters $\chi$ and $v$, and adsorption constant $K_{\text {ads }}$ ). For small $L / B$, isotropic-nematic and/or (depending on $\chi$ ) isotropic liquid-gas coexistence is observed; as $L / B$ increases, coexistence between two different nematic phases appears at low temperature, and liquid-gas equilibrium ceases to be observed for large enough $L / B$; this is understood considering that as aspect ratio increases, the range of stability of the nematic phase becomes larger. Adsorption isotherms are found to significantly deviate from Langmuir behavior, and are strongly affected by ordering and interactions (surface density in the adsorbed layer increases as interaction parameters and ordering increase). Phase coexistence is observed as discontinuous transitions in adsorption isotherms, where adsorption-desorption hysteresis cycles are possible.
\end{abstract}

DOI: 10.1103/PhysRevE.102.062704

\section{INTRODUCTION}

Liquid crystalline (LC) ordering, which involves a "soft," imperfect ordering of particles or molecules while keeping translational mobility, is a widespread phenomenon and can be induced by both excluded-volume effects (entropic) or attractive interactions (enthalpic). Anisotropy in particle's shape or interactions drives the emergence of orientational order, leading to the formation of LC phases. The simplest phase, with twofold symmetry and no positional order, is called nematic, where particles are partially aligned in one direction.

Whenever anisotropic particles or molecules are adsorbed into a surface or strongly confined in a thin space, twodimensional in-plane LC order is possible. Some relevant examples of this are the adsorption of biomacromolecules on membranes or interfaces [1-3], adsorption of rodlike viruses [4], deposition of nanotubes on substrates for electronic components [5], and adsorption of nanocrystalline cellulose at the oil-water interface for food or environmental applications $[6,7]$. Nematic ordering in this case is important not only because it affects adsorption behavior, but also the properties of the system (mechanical and chemical resistance, permeability, in-plane diffusion, electric conductivity, etc.) depend on the structure of the adsorbed layer, affecting its biological or technological function.

In three dimensions (3D), isotropic-nematic transition is a first-order process that is well understood and has been thoroughly studied [8-12]. In-plane LC systems, on the other hand, are different in that, strictly speaking, true long-range order, is not observed in most two-dimensional (2D) systems
[13], because long-wavelength thermal excitations tend to destroy spatial correlations and consequently they do not survive on the macroscopic scale. On a microscopic length scale, correlations decay algebraically as opposed to the exponential decay observed in the disordered phase. Although in systems with nonseparable potentials long-range order cannot be excluded a priori, many systems of this type are observed to display this "quasi-long range order." Therefore, the observed average degree of ordering exhibits a system-size dependence and it is zero in the thermodynamic limit (infinite system). Nevertheless, local order at a microscopic scale does exist, and mean-field theories, which assume true long-range order, can quantitatively describe some aspects of 2D systems $[14,15]$. Additionally, physical systems with 2D order like membranes or confined films, adsorbed monolayers, or interacting multilayers, are actually "quasi-2D systems" as fluctuations or interactions in the third dimension are present. In these systems, true long-range order is, in principle, possible.

Several theoretical approaches, both molecular simulations and mean-field theories, considering hard-core excluded volume effect or attractive interactions, have been developed to study nematic 2D systems. The quasinematic phase with algebraic order is predicted for high surface density [13-16], in a range that becomes broader as the aspect ratio of the particles increases. For hard-core systems with no attractive interactions, the transition from isotropic to quasinematic phase is continuous or second order. Mean-field theories, as mentioned before, rely on the approximation of long-range order, but despite this they reproduce the order-disorder transition, the qualitative behavior of the order parameter, and the equation 
of state fairly well [14]. For interacting systems, it was found that the transition is second order for soft potentials, but becomes first order when the interaction potential is sharp and narrow [17].

There are a few works that considered adsorption behavior in 2D nematic systems, although in very simplified cases: Almeida and Wiegel [18] used a mean field Onsager-type theory and Khandar and Barma [19] performed Monte Carlo simulations of needles considering evaporation and deposition. Both works considered only hard-core excluded-volume effects, in the limit of long, infinitely thin particles. They both found that increasing the pressure or activity in the $3 \mathrm{D}$ gas or solution phase increases the amount of adsorbed particles; the onset of 2D nematic order is observed at the same surface density of systems with conserved number of particles, and nematic order favors adsorption as compared to a disordered state, which produces an abrupt change in the slope of the isotherm at the ordering transition.

In a previous work (part I of the series) [20], a generalized van der Waals theory for LC order in a system of elongated, interacting particles confined to two dimensions was developed. This model combined scaled-particle and Maier-Saupe theories. Order-disorder transitions and equation of state were analyzed as a function of aspect ratio and interaction parameters, and it was observed that these variables strongly affect phase behavior. Inflection points were detected in the equation of state (pressure vs density curves), indicating the presence of phase coexistence. In the present work, that study is extended, using this theory to describe quantitatively phase coexistence, which can involve different types of phases: isotropic-nematic coexistence (IN), which is just a first-order ordering transition, but also isotropic-isotropic (II), and nematic-nematic (NN), which involves coexistence of two phases of equal nature, but with different density (and different degree of ordering in the case of NN); these also could be thought as liquid-gas (LG) transitions. Additionally, adsorption from a solution is analyzed, accounting for the effect of ordering and phase transitions. Full parametric studies in terms of aspect ratio, interaction parameters, and adsorption constant are performed.

\section{MODEL AND METHODS}

\section{A. Free energy and phase ordering}

The model and strategy used for calculating order-disorder transitions was described in part I [20]; here the main points are summarized. The dimensionless free energy for a system of rectangular particles is written by combining scaledparticle theory with Maier-Saupe and van der Waals attractive interactions.

$$
\begin{aligned}
\frac{F_{2 D}}{N R T}= & \ln \phi-\ln (1-\phi)-\phi a_{r}\left(\chi^{\prime}+v^{\prime} S^{2}\right) \\
& +\sigma+\frac{\phi}{1-\phi} \rho+\frac{\mu_{2 D, 0}}{R T}
\end{aligned}
$$

where $\phi$ is the surface density, $a_{r}=B L$ the area of the rectangle, $B$ and $L$ are the width and length of the rectangle, respectively. The first two terms represent translational entropy in the absence of excluded volume, the third term includes isotropic and nematic interactions between particles $\left(\chi^{\prime}\right.$ and $v^{\prime}$ are the isotropic and nematic interaction parameters), the fourth and fifth terms represent the orientational and excluded-volume free energy, and the last term is a reference chemical potential of the rectangles in isotropic state in 2D. Particle-surface interactions are not included explicitly, but they are implicit in this last term. In addition, particle-particle interactions can be mediated or affected by the surface. Moreover, there can be particle-particle interactions that are originated by the surface and do not exist in a 3D system (for example, if the particles produce an elastic deformation on the surface, the deformation fields of different particles can interact [16]). Orientational and excluded volume terms, $\sigma$ and $\rho$, are given by

$$
\begin{gathered}
\sigma=\int_{0}^{\pi} f(\theta) \ln \pi f(\theta) d \theta \\
\rho=\int_{0}^{\pi} d \theta \int_{0}^{\pi} f(\theta) f\left(\theta^{\prime}\right) K\left(\theta-\theta^{\prime}\right) d \theta^{\prime}
\end{gathered}
$$

where $f$ is the orientational distribution function. The kernel $K$ is related to the excluded area between two particles with a relative orientation $\gamma=\theta-\theta^{\prime}$ for hard rectangles it is given by [14]

$$
K(\gamma)=|\cos (\gamma)|+\frac{B^{2}+L^{2}}{2 B L}|\sin (\gamma)| .
$$

Finally, the nematic order parameter is defined as

$$
S=\int_{0}^{\pi} \cos (\theta) f(\theta) d \theta
$$

Isotropic phase is characterized by $S=0$, while in the nematic phase $S>0$.

Functional minimization of the free energy with respect to the orientational distribution function $f$ leads to the equilibrium value of order parameter. This is performed numerically, by expanding the distribution function and the kernel in basis functions; for details see part I [20].

It has to be pointed out that this model predicts the existence of another type of ordered phase, the tetratic phase, as described in Ref. [20]. This phase has fourfold symmetry, that is two (perpendicular) symmetry axes: in the nematic phase $f$ has maxima at $\theta=0$ and $\pi$, in the tetratic phase the maxima are located at $\theta=0, \pi / 2$, and $\pi$. This phase is observed for small $L / B$ and $v$ (a square particle with no interactions has no nematic phase, only isotropic and tetratic). In these conditions, no phase coexistence involving LC phases exists (only continuous tetratic-nematic transitions, or II coexistence if $\chi$ is high enough), so the tetratic phase is not relevant for this work.

\section{B. Equilibrium diagrams}

Phase coexistence is calculated by the condition of thermodynamic equilibrium, which is the equality of pressure and chemical potential in each phase. According to their 
thermo-dynamic definitions, these functions are given by

$$
\begin{gathered}
\frac{B^{2}}{R T} P_{2 D}=-\left.\frac{\partial}{\partial A}\left(\frac{B^{2} F_{2 D}}{R T}\right)\right|_{T, N}=\left[\frac{B}{L} \frac{\phi}{(1-\phi)}-\phi^{2}\left(\chi+v S^{2}\right)+\frac{\phi^{2}}{(1-\phi)^{2}} \frac{B}{L} \rho\right], \\
\frac{\mu_{2 D}}{R T}=\left.\frac{\partial}{\partial N}\left(\frac{F_{2 D}}{R T}\right)\right|_{T, A}=1+\frac{\phi}{(1-\phi)}+\ln \frac{\phi}{(1-\phi)}-2 \phi \frac{L}{B}\left(\chi+v S^{2}\right)+\sigma+\left(\frac{2 \phi-\phi^{2}}{(1-\phi)^{2}}\right) \rho+\frac{\mu_{2 D, 0}}{R T},
\end{gathered}
$$

where $A$ is the area of the surface. Here, dimensionless interaction parameters have been defined as $\chi=B^{2} \chi^{\prime}$ and $v=B^{2} v^{\prime}$. For each point of the phase coexistence diagram, equality of pressure and chemical potential of both phases (each one with different values of $\phi$ and $f$ ) is written, in addition with the functional minimization of the free energy with respect to $f$ for each phase. These equations are solved numerically in order to obtain $\phi$ and $f$ in each phase, for given values of $L / B$ and interaction parameters.

In the case of adsorption equilibrium between the surface and a solution, chemical potential of particles in the surface and in the solution must be equal. Parsons-Lee model $[11,12]$ is used for the three-dimensional systems, for cylindrical particles (as a representative $3 \mathrm{D}$ object with a $2 \mathrm{D}$ section of rectangular shape):

$$
\frac{F_{3 D}}{N R T}=\ln \psi+\frac{1}{4}\left(\frac{L}{B}+\frac{\pi+3}{2}+\frac{\pi}{4}\left(\frac{L}{B}\right)^{-1}\right)\left(\frac{4 \psi-3 \psi^{2}}{(1-\psi)^{2}}\right)+\frac{\mu_{3 D, 0}}{R T},
$$

where $\psi$ is the volume fraction of particles in the solution, and $\mu_{3 D, 0}$ is the reference chemical potential of the particles in solution. This model is selected because it is based in Onsager second-virial theory, but includes a correction in the excludedvolume term that extends its validity to higher densities; in this way it is analogous to the $2 \mathrm{D}$ scaled-particle model used in this work. It has to be noted that no interactions are considered in 3D. As interaction parameters will be, in principle, different in solution and in the surface, accounting for interactions in the solution would require extra parameters to be considered in the analysis. Consequently, the results presented in this work are expected to be valid only for relatively low concentrations of particles in the solution (in addition, low concentration means that only isotropic state must be considered).

The chemical potential of particles in the solution is

$$
\frac{\mu_{3 D}}{R T}=\left.\frac{\partial}{\partial N}\left(\frac{F_{3 D}}{R T}\right)\right|_{T, V}=1+\ln \psi+\frac{1}{4}\left(\frac{L}{B}+\frac{\pi+3}{2}+\frac{\pi}{4}\left(\frac{L}{B}\right)^{-1}\right)\left(\frac{4 \psi-3 \psi^{2}}{(1-\psi)^{2}}+\frac{4 \psi-2 \psi^{2}}{(1-\psi)^{3}}\right)+\frac{\mu_{3 D, 0}}{R T} .
$$

By equating Eqs. (7) and (9), the adsorption isotherms are obtained as

$$
\phi=\frac{K_{\mathrm{ads}} \psi \xi}{1+K_{\mathrm{ads}} \psi \xi},
$$

where $K_{\text {ads }}=\exp \left(\mu_{3 D, 0}-\mu_{2 D, 0}\right)$ is the adsorption (or binding) constant and the factor

$$
\xi=\exp \left[\frac{1}{4}\left(\frac{L}{B}+\frac{\pi+3}{2}+\frac{\pi}{4}\left(\frac{L}{B}\right)^{-1}\right)\left(\frac{4 \psi-3 \psi^{2}}{(1-\psi)^{2}}+\frac{4 \psi-2 \psi^{2}}{(1-\psi)^{3}}\right)-\frac{\phi}{(1-\phi)}+2 \phi \frac{L}{B}\left(\chi+v S^{2}\right)-\sigma+\left(\frac{2 \phi-\phi^{2}}{(1-\phi)^{2}}\right) \rho\right]
$$

includes all the deviations from "ideality" (excluded volume, interactions, and ordering). Langmuir adsorption isotherm is obtained from Eq. (10) by disregarding all these nonidealities and making $\xi=1$. In the general case of $\xi \neq 1$, Eq. (10) has to be solved numerically.

\section{RESULTS AND DISCUSSION}

\section{A. Phase coexistence in $2 \mathrm{D}$}

As mentioned in our previous work, several inflection points in the equation of state plots (pressure vs surface density) were observed, indicating different types of phase coexistence (II, IN, and NN). In this section, these phase diagrams will be quantitatively explored in detail.

First, II, or LG, phase coexistence is shown, disregarding phase ordering. Although this is not necessarily the equilibrium state of the system (which in general involves the nematic phase), it could represent a metastable state if ordering is disfavored (for example if translational diffusion is faster than rotational diffusion). Figure 1(a) shows phase coexistence diagrams, in terms of the inverse of a scaled dimensionless temperature, defined as $(L / B \chi)^{-1}$. Increasing $L / B$ displaces the critical point to smaller surface density. Figure 1(b) shows that the value of $\chi^{-1}$ at the critical point increases as a function of aspect ratio; approaching an asymptotic limit of 0.3183 ; the inset shows that $(\chi-0.3183)^{-1}$ vs $L / B$ plotted in log-log scale shows a linear relationship (with small deviations for small $L / B$ ).

Figure 2 shows IN and NN phase coexistence, in the absence of isotropic interactions. Again, phase coexistence is displaced to smaller values of $\phi$ and higher values of $v^{-1}$ as $L / B$ increases. In our previous work it was found that for small values of $v$ (high temperature), the order-disorder transition is continuous, but as the quadrupolar parameter increases, at some point the transition becomes first order; consequently, as observed in Fig. 2, phase coexistence exists only below a given value of $v^{-1}$ which is a function of $L / B$. Moreover, the type of phases involved are also different for different $L / B$ : for relatively short rectangles, only IN coexistence is observed, while for longer rods a $\mathrm{NN}$ coexistence exists in some range 

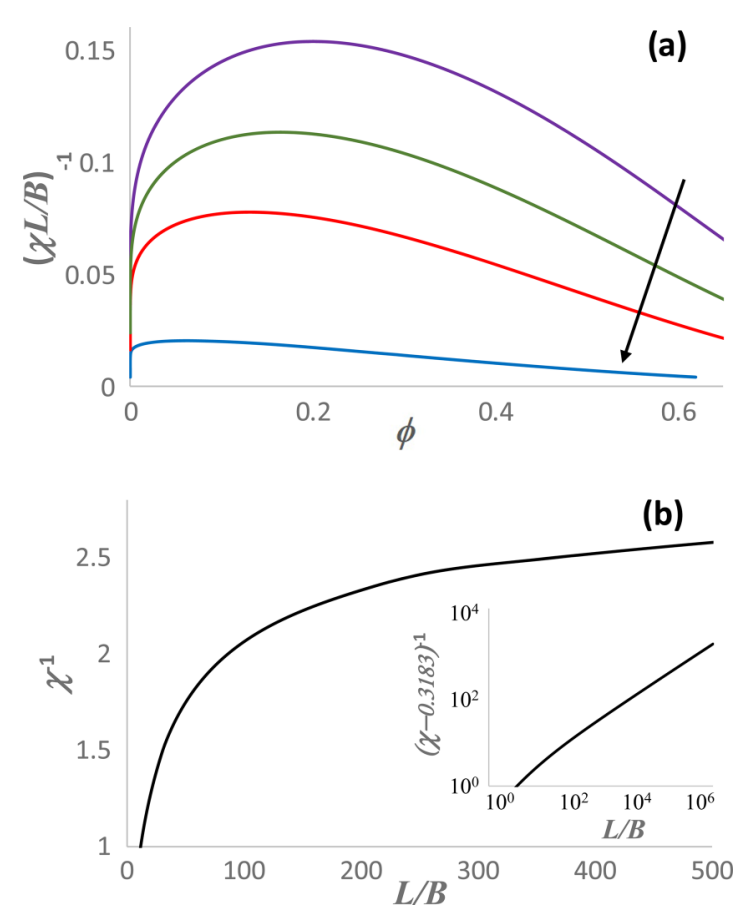

FIG. 1. Phase behavior, disregarding ordering. (a) Coexistence diagrams in terms of scaled dimensionless temperature $(\chi L / B)^{-1}$, as a function of the surface density $\phi$, for different values of $L / B$ (increasing in the direction of the arrow): 1.5 (purple), 6 (green), 14 (red), 100 (blue). Above each curve the system is homogeneous; below the curve it is phase separated in two isotropic phase of different densities (liquid and gas). (b) Value of interaction parameter at the critical point as a function of $L / B$. The inset shows $(\chi-0.3183)^{-1}$ as a function of $L / B$ in $\log -\log$ scale.

of temperature that becomes broader as $L / B$ increases (for low enough temperature, IN equilibrium is always found).

Once the different possible phase equilibria and the effect of interaction parameters have been analysed and understood separately, Fig. 3 shows the complete phase diagrams considering both types of interactions, in terms of the interaction

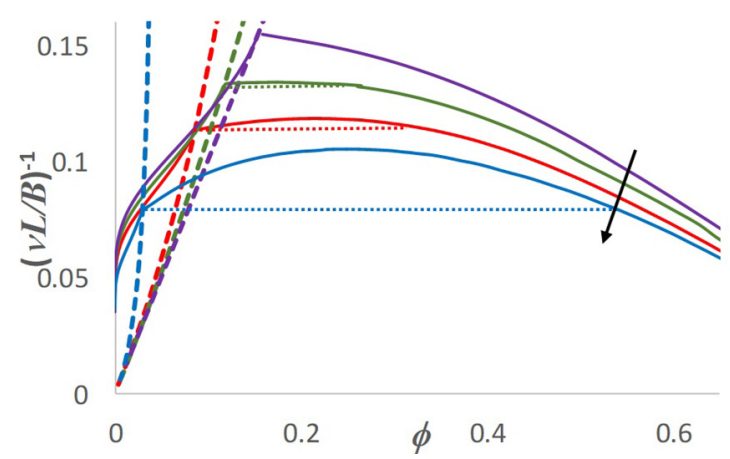

FIG. 2. Phase equilibrium diagrams, as in Fig. 1 but for a system with $\chi=0 . L / B$ increases in the direction of the arrow (same values and color as Fig. 1). Dashed lines represent IN transitions in a homogeneous phase, and dotted horizontal lines represents coexistence of three phases: one isotropic and two nematic. Between the full curves and the dotted lines, two nematic phases coexist; below the dotted line there is IN coexistence. parameter ratio $r=\chi / \nu$, and where temperature is made dimensionless with respect to the nematic quadrupolar interaction parameter. Besides the general trend that increasing $r$ displaces phase diagrams to higher values of $v^{-1}$, the behavior is quite different for different values of $L / B$. II equilibrium is observed only for relatively small values of $L / B$, while for longer rectangles it remains always "buried" below IN or NN equilibria. Even for small $L / B$ the shape of the phase diagrams is different for large $r$; for $L / B=6$ there is a clearly distinguishable coexistence region between nematic and high-density isotropic phase (nematic-isotropic liquid) above the triple line, while for $L / B=1.5$ only a nematicgas coexistence is observed (there is actually a very small nematic-liquid coexistence region, but it is not noticeable). In addition, as mentioned before, NN coexistence was observed for $L / B=6$ or larger in the absence of isotropic interactions; as $r$ increases, this ceases to exist and only IN coexistence is observed for intermediate $L / B$, but for the largest rectangles analysed, $\mathrm{NN}$ coexistence remains for every value of $r$. This can be understood by considering that increasing $L / B$ broadens the density range where the nematic phase is stable; consequently II or IN equilibria dominate for short particles and NN coexistence for large $L / B$.

\section{B. Adsorption equilibrium}

In this section, generalized van der Waals theory is used to describe adsorption of cylindrical particles from an isotropic solution with no interactions ("dilute" regime) to a surface. Adsorption curves are constructed up to the solution isotropicnematic transition point. First, the effect of $K_{\text {ads }}$ on adsorption isotherms is shown in Fig. 4 for different values of $L / B$. Logarithmic scale is used to better appreciate the low-concentration regime. The effect is relatively similar for every value of $L / B$. For comparison, Fig. 4 shows also the Langmuir adsorption isotherm. It can be noted that the system strongly deviates from Langmuir behavior except at very low concentration. It is interesting to note that deviations from Langmuir behavior do not have the same sign for every value of $K_{\text {ads }}$ : adsorption is stronger than Langmuir for small $K_{\text {ads }}$ and weaker for large $K_{\text {ads }}$. For an intermediate value of $K_{\text {ads }}$ of about 4 , deviations from Langmuir adsorption are smallest for all $L / B$ and the behavior can be reasonably approached by this model in all the range of existence of the isotropic phase, but for different values of $K_{\text {ads }}$, as mentioned before, deviations become very strong even for small concentrations. This can be understood as follows: small $K_{\text {ads }}$ implies little adsorption so the adsorbed monolayer is "more diluted" than the solution (this is in relative terms; a direct comparison of the values of $\psi$ and $\phi$ does not necessarily makes direct sense). Excluded-volume interactions, which always decrease entropy and increase free energy, are then more significant in the solution, and consequently adsorption is favored (with respect to a system with no excluded-volume interactions). Mathematically, the factor $\xi$ in Eq. (10) is larger than 1 for small $K_{\text {ads }}$, which increases $\phi$ for given $\psi$ and $K_{\text {ads }}$. On the other hand, large $K_{\text {ads }}$ favors higher concentration in the adsorbed layer, $\xi<1$, and the opposite is observed; for $K_{\mathrm{ads}}=4$ deviations in both phases are more or less balanced and $\xi$ is close to 1 . Note that this is already observed for very low concentrations where Onsager 

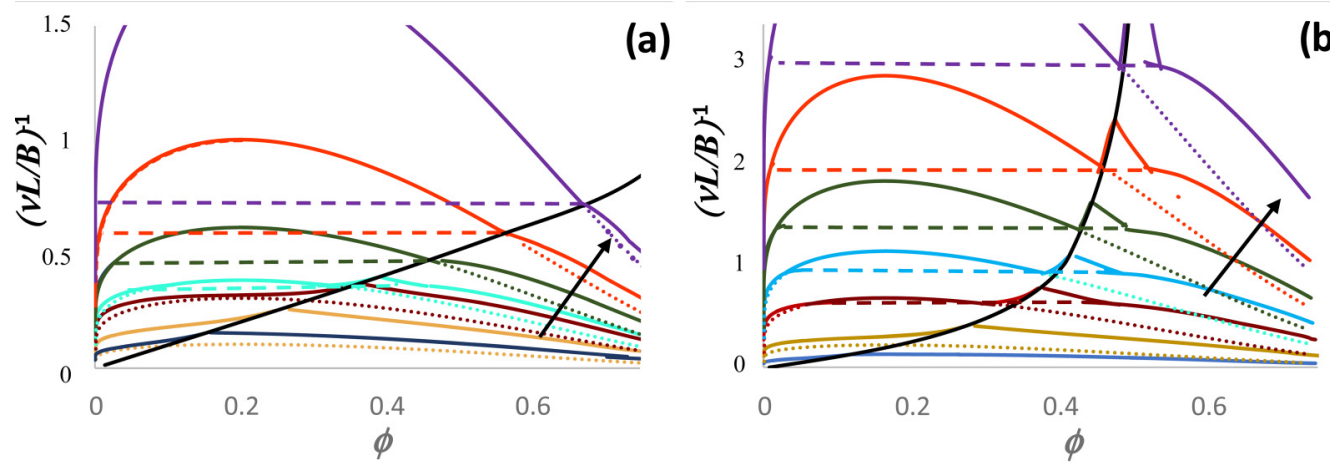

(b)
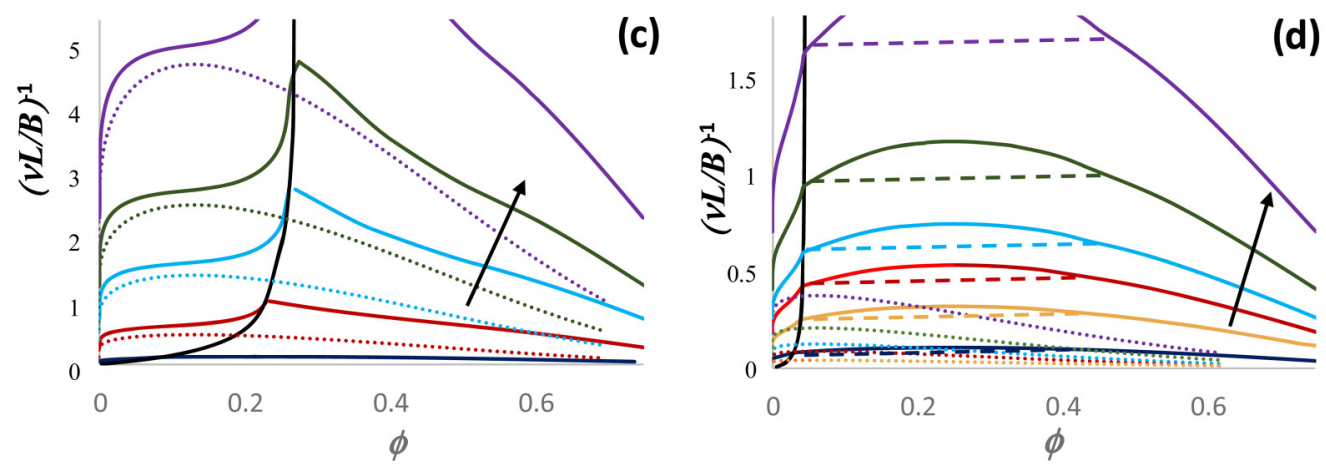

FIG. 3. Phase diagrams, as in Fig. 1, for systems with both isotropic and anisotropic interactions. The parameter $r=\chi / \nu$ increases in the direction of the arrows. The values of $L / B$ and $r$ are (a) $L / B=1.5, r=0,0.66,2,2.5,4,6.5,12$; (b) $L / B=6, r=0,2,6,10,16,25,40$; (c) $L / B=14, r=0,6,18,32,60$; and (d) $L / B=100, r=0,2,4,6,10,18$. Thick black lines represent IN transitions in a homogeneous phase, dotted lines are metastable II phase coexistence regions, and dashed horizontal lines represent coexistence of three phases: one isotropic and two nematic or two isotropic and one nematic. Different regions of the phase diagrams are the same as described in previous figures.
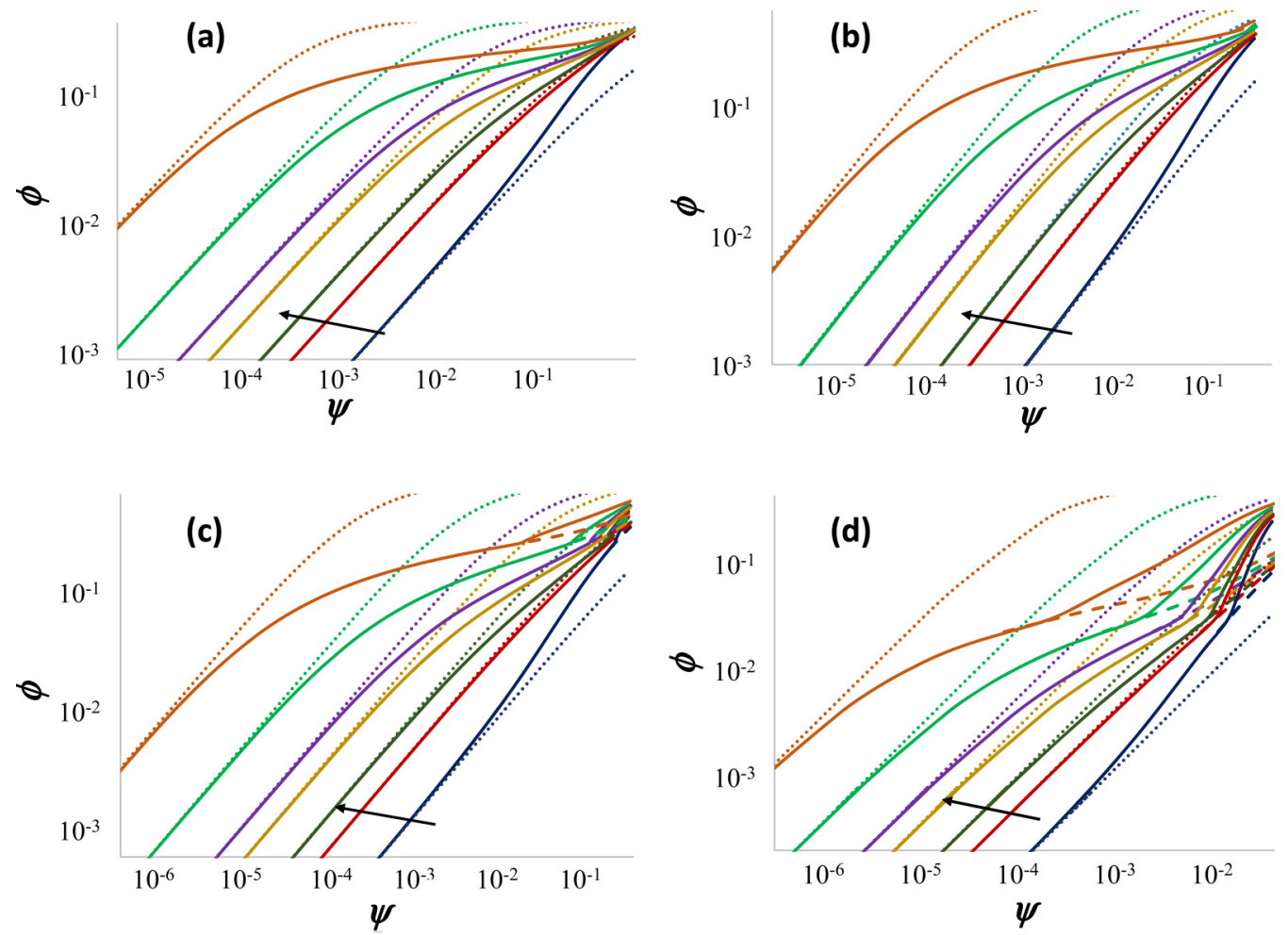

FIG. 4. Adsorption isotherms for systems with no interactions. $L / B=1.5$ (a), 6 (b), 14 (c), and 100 (d), representing the surface density of the adsorbed $\phi$ particles as a function of their concentration in solution $\psi$. The different values of $K_{\text {ads }}$, increasing in the direction of the arrows, are 1, 4, 8, 25, 50, 250, and 3000. Dashed lines indicate the metastable isotropic state, and dotted lines represent Langmuir isotherms. 

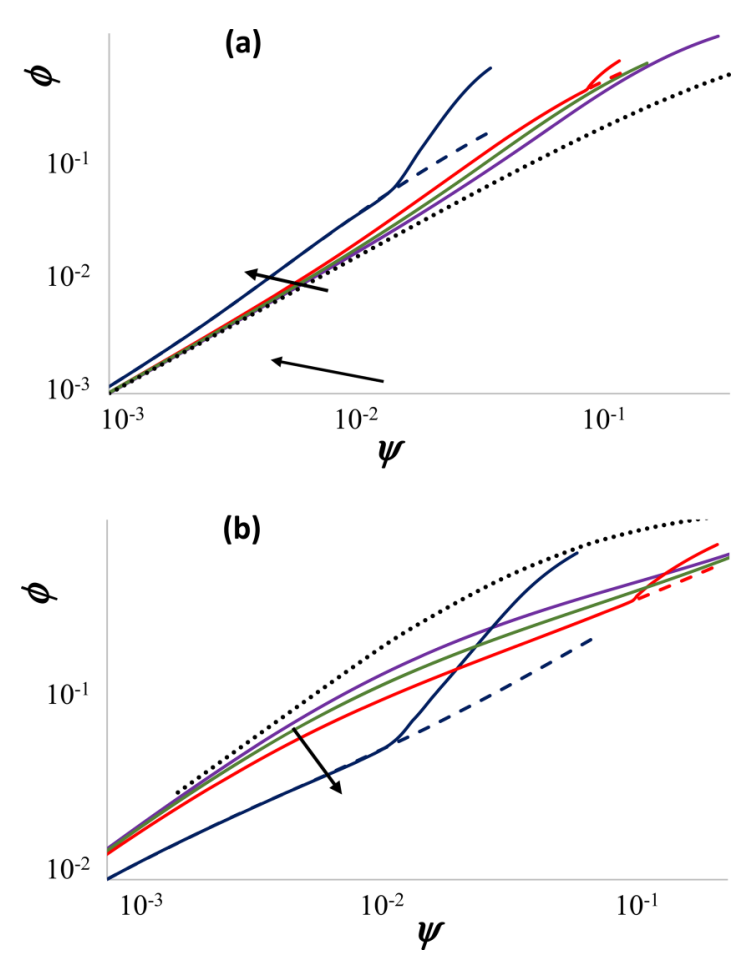

FIG. 5. Adsorption isotherms for $K_{\text {ads }}=1$, with no interactions, for different values of $L / B: 1.5,6,14$, and 100 (increasing as indicated by the arrow). Dashed lines indicate the metastable isotropic state, and dotted lines represent Langmuir isotherms.

(second-virial) approximations are accurate, so this value is not expected to be strongly model-dependent (provided that the model captures at least a second-virial approximation).
In addition, it can be observed that nematic order increases adsorption. This is caused by the fact that the free energy, and thus the chemical potential, in the equilibrium nematic phase is lower than in the isotropic phase.

Figure 5 shows the effect of $L / B$ for given values of $K_{\text {ads }}$, one smaller and one larger than 4 . It can be observed that increasing $L / B$ increases deviation from Langmuir behavior in the isotropic phase; as discussed before, excluded-volume effects, which are more important as $L / B$ increases, cause these deviations. This means that as $L / B$ increases, adsorption is favored if $K_{\text {ads }}<4$, while it is disfavored for larger $K_{\text {ads }}$. As observed before, the effect of nematic ordering is to increase adsorption. As nematic ordering is determined by excluded volume, the effect of $L / B$ on adsorption in the nematic state is very significant.

In Fig. 6, the effect of $v$ is shown for different $L / B, K_{\mathrm{ads}}=$ 1 , and $\chi=0$. As the quadrupolar interaction increases, NI transition is produced at smaller concentration, which, as mentioned before, increases adsorption. Within the nematic phase range, larger $v$ produce larger increases in the surface density of adsorbed phase, as expected due to the fact that nematic interactions are attractive and lower the free energy (it has to be remembered that no interactions in the isotropic phase are considered). Increasing $v$ not only shifts the curves to lower $\psi$ and larger $\phi$, but also changes the shape of the curves: the slope of the curves at the transition increases, until the curves develop a vertical inflection point. This is the value of $v$ at which, as analyzed in the previous section, the IN transition become first order or discontinuous. For larger values of the quadrupolar interaction parameter, there is a discontinuity seen as a vertical stepwise change in the concentration of adsorbed particles at the IN transition. In this
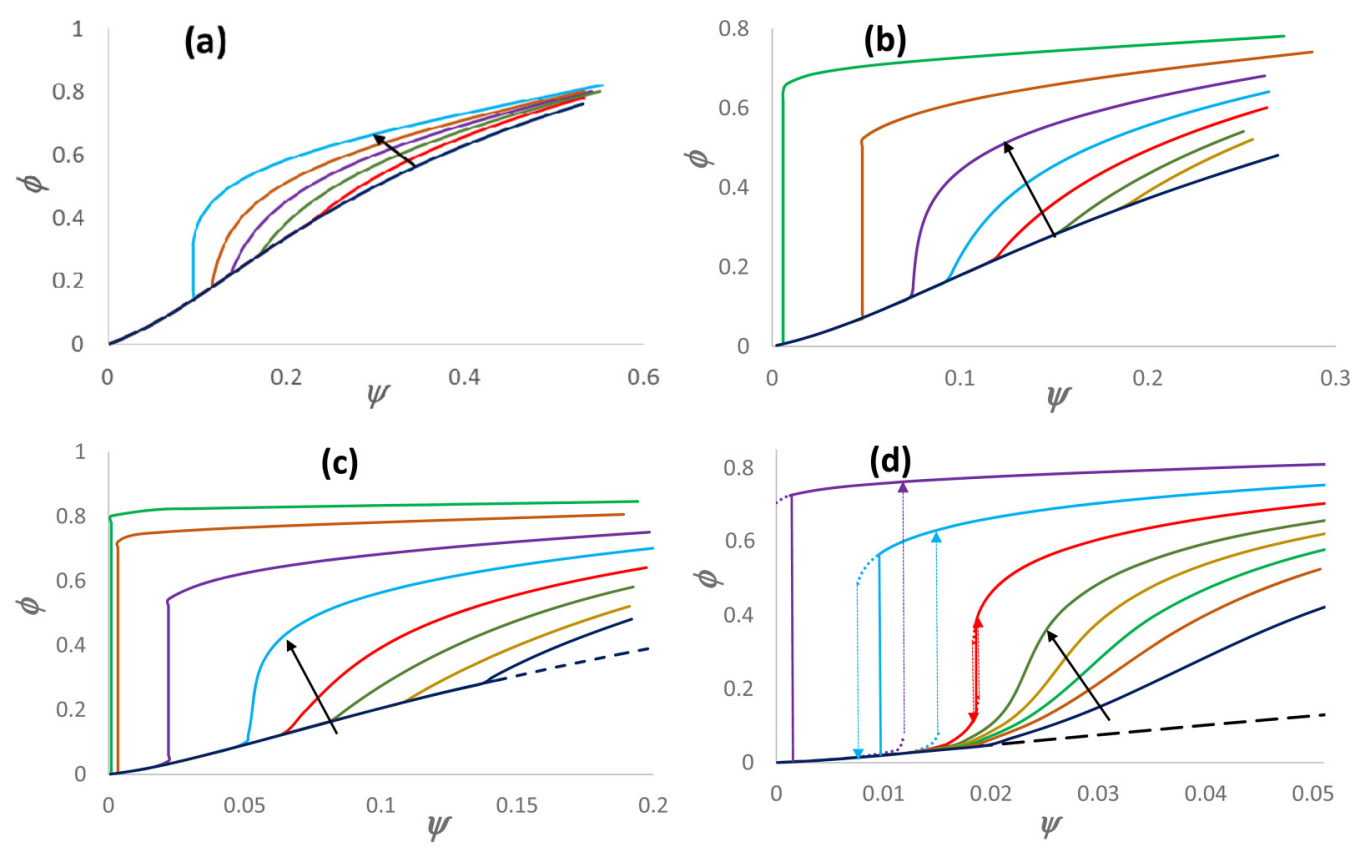

FIG. 6. Adsorption isotherms, for $K_{\text {ads }}=1$ and $\chi=0$, and $L / B=1.5$ (a), 6 (b), 14 (c), and 100 (d). The quadrupolar interaction parameter increases in the direction of the arrow, as follows: (a) $0,1.667,2.333,3,3.667$, and 4.667 ; (b) $0,0.25,0.375,0.583,0.833$, 1.167, 1.667, and 2.5; (c) $0,0.0858,0.215,0.358,0.572,0.858,1.43$, and 2.15; and (d) $0,0.03,0.045,0.06,0.075,0.1,0.14$, and 0.22 . In (c) and (d), dashed lines indicate the metastable isotropic phase. In (d) dotted lines represent metastable high-and low-concentration nematic phases, indicating possible hysteresis. Hysteresis cycles when increasing or decreasing concentration are represented by dotted thin arrows. 

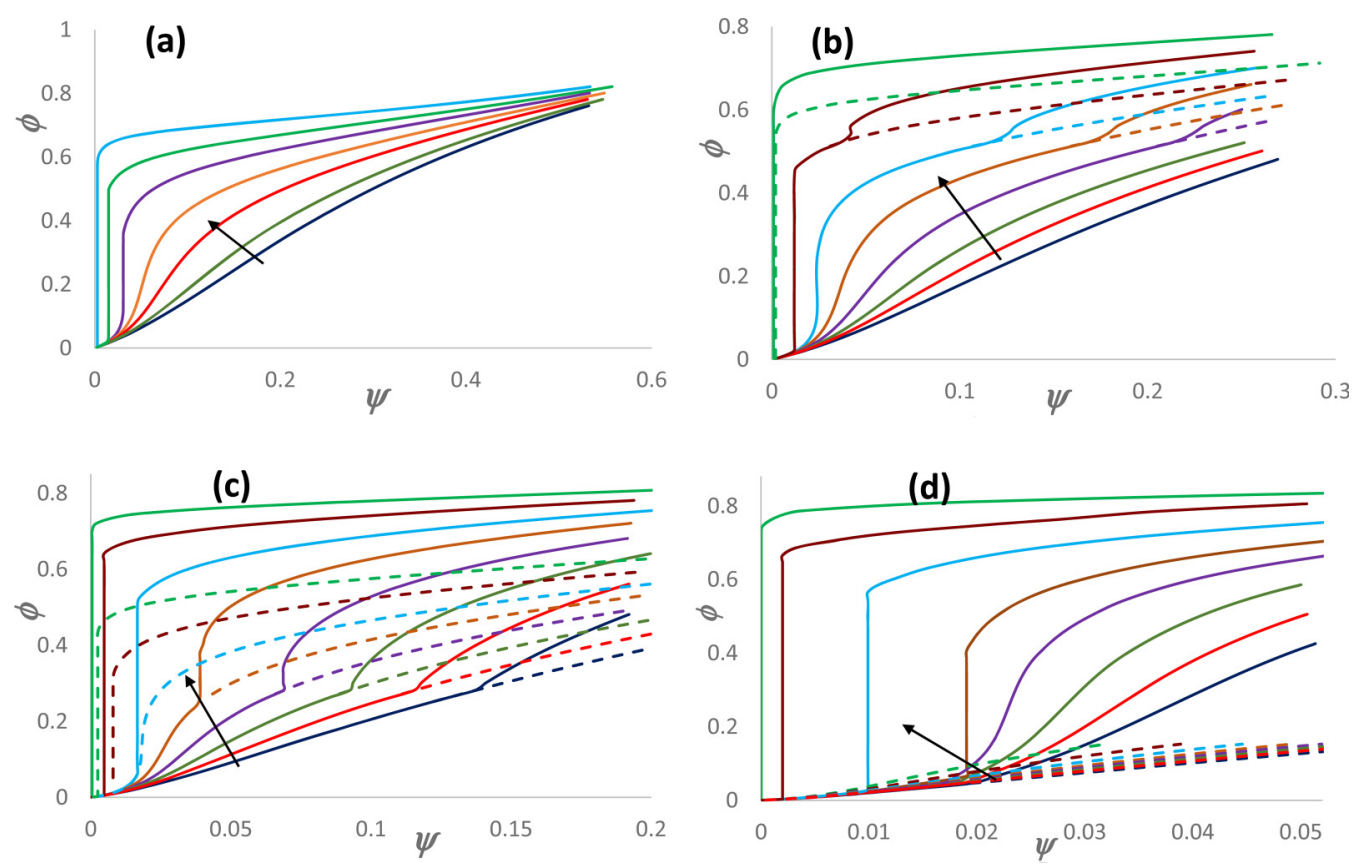

FIG. 7. Adsorption isotherms, for $K_{\mathrm{ads}}=1$ and $v=0$, and $L / B=1.5$ (a), 6 (b), 14 (c), and 100 (d). The isotropic interaction parameter increases in the direction of the arrow, as follows: (a) 0, 0.833, 2.22, 3.16, 3.33, 4.66, 6, and 8; (b) 0, 0.25, 0.5, 0.83, 1.16, 1.5, 2, and 2.67; (c) $0,0.179,0.358,0.537,0.715,0.9,1.15$, and 1.43 ; and (d) $0,0.025,0.05,0.07,0.1,0.14,0.19$, and 0.22 . Dashed lines indicate the metastable isotropic phase.

case, adsorption-desorption hysteresis could be expected, as both low- and high-concentration adsorbed phases can exist in a metastable state in some density range beyond the transition point; this is shown in Fig. 6(d). Hysteresis effect, that is, the range of existence of metastable phases, increases as $v$ increases; at some point the high-concentration phase can remain metastable even when $\psi$ decreases to 0 .

Finally, Fig. 7 shows the effect of isotropic interactions $\chi$, in system with no quadrupolar interactions and $K_{\mathrm{ads}}=1$. The effect of $\chi$ is similar to $\nu$, in that it favors adsorption by decreasing the free energy of adsorbed particles. Again, different types of phase coexistence can be induced, producing the respective discontinuities in concentration; even two of these jumps can be observed in some cases, corresponding to temperatures where II and IN coexistence is observed in different ranges of surface density. Again, hysteresis could be observed in these cases (not shown in the figure). In addition, as mentioned in the previous section, II metastable phase coexistence can exist when IN coexistence is the equilibrium state; this could lead, for example, to a two-step adsorption, when $\psi$ is increased beyond the transition point: first the high- $\phi$ isotropic would be formed, which can become nematic in a second step. This two-step phase transition process, consistent with Oswald rule of phase transitions, was analyzed for nematic systems with a phase-field model, and depends on the relative values of diffusional and ordering mobilities [21,22].

\section{CONCLUSIONS}

The generalized van der Waals equation for a system of rodlike particles in $2 \mathrm{D}$ developed in a previous work was used to calculate phase coexistence diagrams and adsorption isotherms, analyzed as a function of the model parameters (aspect ratio $L / B$, isotropic and anisotropic interaction parameters $\chi$ and $v$, and adsorption constant $K_{\text {ads }}$ ). A rich phase behavior was observed, including II, IN, and NN coexistence; in addition, and as already observed in part I [20], for high temperatures IN transition is continuous with no phase coexistence. Not only does the temperature range for phase coexistence increase with $L / B$, but also the phase diagrams change qualitatively: regarding ordering transitions, only IN is observed for short rods, for every value of $r$, only hightemperature-NN+low-temperature-IN for long rods, and for intermediate $L / B, \mathrm{NN}+\mathrm{IN}$ is observed for low $r$ and only IN for high $r$. In addition, equilibrium involving II coexistence (liquid-gas transition) only exists for short and intermediate rods $(L / B=1.5$ and 6$)$, while for larger aspect ratio (14 and 100) II phase coexistence always remains buried below IN or NN coexistence diagrams (indicating that II could be a metastable state, but does not represent absolute equilibrium).

Adsorption behavior in general deviates strongly from Langmuir isotherms even at small concentration, with positive and negative deviation for large and small values of $K_{\mathrm{ads}}$, respectively. An exception for this was observed when $K_{\text {ads }}$ is in the vicinity of 4, where positive and negative deviations (which are related to excluded-volume effects in solution and in the adsorbed monolayer) balanced each other and the behavior was very close to Langmuir adsorption (in the isotropic phase). As interaction parameters increase, the amount of adsorbed particles increase due to a decrease in free energy, and for the same reason nematic ordering favors adsorption as compared to an isotropic system. 2D phase coexistence was observed as vertical discontinuities in the adsorption isotherms, with the possibility of hysteresis cycles. A two-step adsorption is possible when IN (or NN) and metastable II coexistence are simultaneously present. 
Although the model is quite simple, it provides a complete description of complex phase and adsorption diagrams, and shows that the geometrical and interaction parameters have a strong effect on the behavior of the system and cannot be neglected.

\section{ACKNOWLEDGMENTS}

This work was funded by the National Research Council (CONICET), the Agency for Promotion of Science and Technology (ANPCyT), and University of Mar del Plata (UNMDP).
[1] S. Yamaguchi, D. Huster, A. Waring, R. I. Lehrer, W. Kearney, B. F. Tack, and M. Hong, Biophys. J. 81, 2203 (2001).

[2] A. Yu. Antipina and A. A. Gurtovenko, J. Phys. Chem. B 119, 6638 (2015).

[3] S. Jordens, K. Schwenke, I. Usov, E. Del Gado, and R. Mezzenga, Soft Matter 12, 1830 (2016).

[4] P. J. Yoo, K. T. Nam, J. Qi, S. K. Lee, J. Park, A. M. Belcher, and P. T. Hammond, Nat. Mater. 5, 234 (2006).

[5] M. Burghard, G. Duesberg, G. Philipp, J. Muster, and S. Roth, Adv. Mater. 10, 584 (1999).

[6] C. Gómez H., A. Serpa, J. Velásquez-Cock, P. Gañán, C. Castro, L. Vélez, and R. Zuluaga, Food Hydrocoll. 57, 178 (2016).

[7] Z. Chen, C. An, J. Yin, E. Owens, K. Lee, K. Zhang, and X. Tian, J. Hazard. Mater. 402, 123464 (2021).

[8] L. Onsager, Ann. N. Y. Acad. Sci. 51, 627 (1949).

[9] W. Maier and A. Saupe, Z. Naturforsch. A 15, 287 (1960).

[10] W. Maier and A. Saupe, Z. Naturforsch. A 14, 882 (1959).
[11] J. D. Parsons, Phys. Rev. A 19, 1225 (1979).

[12] S.-D. Lee, J. Chem. Phys. 87, 4972 (1987).

[13] D. Frenkel and R. Eppenga, Phys. Rev. A 31, 1776 (1985).

[14] H. Schlacken, M. J. Mogel, and P. Schiller, Mol. Phys. 93, 777 (1998).

[15] J. A. Cuesta and D. Frenkel, Phys. Rev A 42, 2126 (1990).

[16] S. Kumari, F. Ye, and R. Podgornik, J. Chem. Phys. 153, 144905 (2020).

[17] R. L. C. Vink, Phys. Rev. Lett. 98, 217801 (2007).

[18] P. F. F. Almeida and F. W. Wiegel, J. Theor. Biol. 238, 269 (2006).

[19] M. D. Khandkar and M. Barma, Phys. Rev. E 72, 051717 (2005).

[20] M. V. Zonta and E. R. Soulé, Phys. Rev. E 100, 062703 (2019).

[21] E. R. Soulé, C. Lavigne, L. Reven, and A. D. Rey, Phys. Rev. E 86, 011605 (2012).

[22] E. R. Soulé and A. Rey, Europhys. Lett. 86, 46006 (2009). 